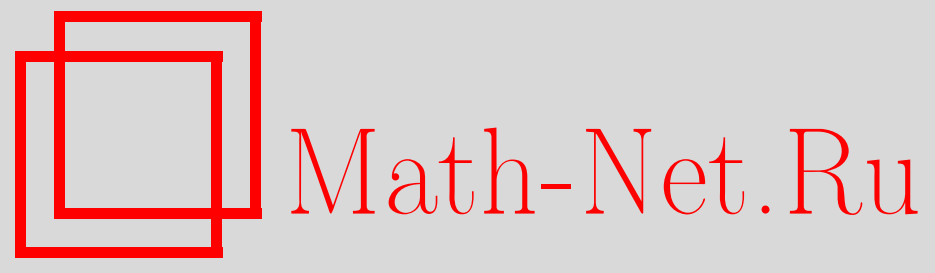

Л. В. Степанова, Е. М. Адылина, Поле напряжений у вершины трещины при смешанном нагружении в условиях плоского напряженного состояния, Вестн. Сам. гос. техн. ун-та. Сер. Физ.-мат. науки, 2014, выпуск 1(), 109-124

DOI: https://doi.org/10.14498/vsgtu1297

Использование Общероссийского математического портала MathNet.Ru подразумевает, что вы прочитали и согласны с пользовательским соглашением

http: //www.mathnet.ru/rus/agreement

Параметры загрузки :

IP : 35.173 .219 .12

26 апреля 2023 г., 05:38:25

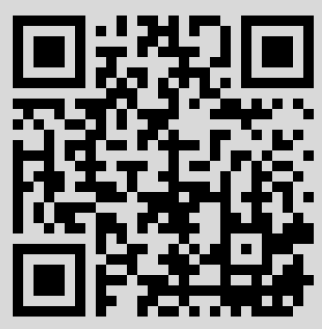


УДК 539.376

\title{
ПОЛЕ НАПРЯЖЕНИЙ У ВЕРШИНЫ ТРЕЩИНЫ ПРИ СМЕШАННОМ НАГРУЖЕНИИ В УСЛОВИЯХ ПЛОСКОГО НАПРЯЖЕННОГО СОСТОЯНИЯ
}

\author{
Л. В. Степанова, Е. М. Адылина \\ Самарский государственный университет, \\ Россия, 443011, Самара, ул. Академика Павлова, 1.
}

\begin{abstract}
Приводится асимптотическое решение задачи о смешанном (двухосном) нагружении образца с трещиной в условиях плоского напряженного состояния с нелинейными определяющими уравнениями материала, удовлетворяющими дробнолинейному закону теории установившейся ползучести. Определены поля напряжений и скоростей деформащий ползучести у вершины трещины в условиях смешанного нагружения. Вид смешанного нагружения задается параметром смешанности нагружения, который изменяется от 0, что отвечает чистому сдвигу, до 1, что соответствует чистому нормальному отрыву. Получены аналитические представления компонент тензора напряжений и скоростей деформаций ползучести в непосредственной окрестности вершины трещины в полном диапазоне смешанных форм дебормирования: от нормалъного отрыва до поперечного сдвига.
\end{abstract}

Ключевые слова: смешанное (двухосное) нагружение, асимптотический анализ, поля напряжений у вершины трещины, аналитическое представление напряжений.

Введение. Смешанное нагружение трещины (нормальный отрыв и поперечный сдвиг) в условиях плоского напряженного состояния. Вопросы смешанного нагружения элементов конструкций с трещинами и угловыми вырезами являются предметом пристального исследования научного сообщества в самое последнее время [1-19]. В [6] разработан метод и приведены результаты вычислений упругопластических коэффициентов интенсивности напряжений в полном диапазоне смешанных форм деформирования от нормального отрыва до чистого сдвига. Кроме этого, в [6] рассмотрено состояние произвольно ориентированной прямолинейной трещины в виде математического разреза при двухосном нагружении различной интенсивности. Решение основано на использовании уравнения совместности деформаций, представленное через потенциал напряжений Әри и его производные. В [6] предполагалось, что поведение упругопластического материала соответствует модели РамбергаОсгуда. На основе выполненных расчетов установлен характер влияния вида смешанных форм нагружения и пластических свойств материала, описываемых показателем деформационного упрочнения.

ISSN: 2310-7081 (online), 1991-8615 (print); doi: http://dx.doi.org/10.14498/vsgtu1297 (C) 2014 Самарский государственный технический университет.

Образец цитирования: Л.В.С теп ан ова, Е.М.А дылина, "Поле напряжений у вершины трещины при смешанном нагружении в условиях плоского напряженного состояния" // Вестн. Сам. гос. техн. ун-та. Сер. Физ.-мат. науки, 2014. № 1(34). С. 109-124. doi: $10.14498 /$ vsgtu1297.

Сведения об авторах: Лариса Валентиновна Степанова (д.ф.-м.н., доц.), профессор, каф. математического моделирования в механике. Екатерина Михайловна Адылина, аспирант, каф. математического моделирования в механике.

E-mail addresses: 1st@ssu.samara.ru (L.V. Stepanova, Corresponding author), kateadulina@mail.ru (E.M. Adylina) 
В [7] предложен метод и приведены результаты расчетов упругих параметров смешанности и скорости выделения энергии в трехмерной постановке задачи при сочетании форм смещения поверхностей трещины по типу отрыва, сдвига и среза. Изучено состояние произвольно ориентированной несквозной полуэллиптической трещины при двухосном нагружении различной интенсивности. Процедура вычислений построена на модификации аналитического решения корректирующими функциями учета выхода вершин полуэллиптической трещины на свободную поверхность образца. На основе выполненных расчетов установлен характер влияния вида смешанных форм нагружения и формы в плане несквозного дефекта на изменение параметров вдоль криволинейного фронта трещины. В [8] рассмотрены подходы и методы нахождения напряженно-деформированного состояния у вершины трещины, ее траектории и скорости ее роста при смешанных формах двухосного нагружения. В [9] на основе численного решения задач в упругой и физически нелинейной постановке для крестовидного образца, пластины с центральной наклонной трещиной и компактного образца с односторонним боковым надрезом получены зависимости между обобщенными параметрами состояния для смешанных форм деформирования. Работы [10,11] посвящены методикам усталостных испытаний стальных крестообразных образцов с поверхностной трещиной при двухосном нагружении. Предложен образец и приспособление для осуществления в рабочей части образца смешанного деформирования. В этих работах показано, что предложенная методика экспериментального исследования дает возможность оценить восприимчивость различных материалов к двухосному нагружению при развитии усталостных трещин. Исследование характеристик циклической трещиностойкости конструкционной стали в условиях смешанного нагружения выполнено в [12], где на основе численных расчетов напряженно-деформированного состояния в крестообразном образце с центральной трещиной определены значения Т-напряжений и К-тарировочных функций. Экспериментально установлено влияние вида напряженного состояния на скорость роста трещины при двухосном деформировании.

В [13] получено приближенное решение задачи о трещине, находящейся под одновременным действием растягивающей и сдвиговой нагрузки, в материале, подчиняющемся дробно-линейному закону теории установившейся ползучести в предположении реализации плоского деформированного состояния. В [14] на основе поляризационно-оптического метода (метода фотоупругости) выполнено экспериментальное исследование напряженно-деформированного состояния в окрестности вершины трещины в образцах с надрезами, находящимися в условиях смешанного нагружения. Проведенное экспериментальное исследование позволило определить коэффициенты интенсивности напряжений в асимптотическом разложении полей напряжений и перемещений в окрестности вершины трещины, а также найти коэффициенты высших приближений в полном асимптотическом разложении М. Уильямса. В [15] получено асимптотическое решение задачи определения напряженно-деформированного состояния и поля сплошности в окрестности вершины трещины в образце, находящемся в условиях смешанного деформирования. На основании автомодельного представления решения и гипотезы о формировании области полностью дефрагментированного материала вблизи вершины трещины получено распределение напряжений, скоростей деформаций и сплошности у стационарной трещины в среде с поврежденностью в полном диапазоне смешанных форм деформирования (от чистого сдвига до нормального отры- 
ва). Построены высшие приближения в асимптотических разложениях полей напряжений, скоростей деформаций ползучести и сплошности. Тем не менее многие вопросы смешанного нагружения элементов конструкций с трещинами остаются открытыми и заслуживают отдельного обсуждения. Например, реализация подхода, развитого в [15-19] для трещин антиплоского сдвига, нормального отрыва и поперечного сдвига, а также для смешанных форм (тип I/I) для плоского деформированного состояния, для случая плоского напряженного состояния требует детального изучения поля напряжений у вершины трещины, поскольку в случае плоского напряженного состояния угловые распределения компонент тензора напряжений у границ рассматриваемого отрезка интегрирования претерпевают резкие изменения, которые сложно учесть в рамках численного анализа. Особенно ценным в данным случае является наличие аналитического представления угловых распределений у кончика трещины, что и является предметом настоящего исследования. Следует отметить, что задачи определения плоского напряженного состояния для тел с вырезами недостаточно изучены и активно исследуются в последнее время [20-22].

Целью настоящей работы является изучение полей напряжений и скоростей деформации ползучести вблизи вершины трещины в условиях смешанного нагружения в материале, подчиняющемся дробно-линейному закону теории установившейся ползучести. В работе получено приближенное решение задачи о трещине, находящейся под одновременным действием растягивающей и сдвиговой нагрузок в материале, подчиняющемся дробно-линейному закону теории установившейся ползучести в предположении реализации плоского напряженного состояния. Вид нагружения характеризуется параметром смешанности нагружения [2, 23-27] и определяется как соотношение окружной и сдвиговой компонент тензора напряжений на линии продолжения трещины:

$$
M^{p}=\frac{2}{\pi} \operatorname{arctg}\left|\lim _{r \rightarrow 0} \frac{\sigma_{\theta \theta}(r, \theta=0)}{\sigma_{r \theta}(r, \theta=0)}\right| .
$$

Параметр смешанности нагружения принимает нулевое значение для трещины поперечного сдвига и значение, равное единице, для чистого растяжения с областью значений из интервала $0<M^{p}<1$.

Постановка задачи о смешанном нагружении (нормальный отрыв и поперечный сдвиг) трещины в условиях плоского напряженного состояния. Задача определения напряженно-деформированного состояния у вершины трещины в нелинейных материалах была и остается одной из актуальных проблем механики разрушения $[1,2]$. В настоящей работе рассматриваются поля напряжений и скоростей деформаций ползучести вблизи вершины трещины в материале с дробно-линейным законом теории установившейся ползучести при смешанном нагружении (отрыв и поперечный сдвиг) образца с трещиной в предположении о реализации плоского напряженного состояния. Развитый в нелинейной механике разрушения подход, основанный на асимптотическом представлении механических полей у кончика трещины, может быть успешно применен и к трещине смешанного типа в условиях плоского напряженного состояния. С этой целью мы обратимся к квазилинейным уравнениям теории установившейся ползучести:

$$
\dot{\varepsilon}_{i j}=(3 / 2) f\left(\sigma_{e}\right) s_{i j} / \sigma_{e},
$$

где $f\left(\sigma_{e}\right)=B \sigma_{e} /\left(\sigma_{b}-\sigma_{e}\right), B, \sigma_{b}$ - материальные константы, определяемые 
экспериментально; $s_{i j}$ - девиатор тензора напряжений, $\sigma_{e}=\left(3 s_{i j} s_{i j} / 2\right)^{1 / 2}-$ интенсивность касательных напряжений. Сформулируем систему уравнений равновесия

$$
r \sigma_{r r}, r+\sigma_{r \theta, \theta}+\left(\sigma_{r r}-\sigma_{\theta \theta}\right)=0, \quad r \sigma_{r \theta, r}+\sigma_{\theta \theta, \theta}+2 \sigma_{r \theta}=0
$$

и условие совместности деформаций

$$
2\left(r \dot{\varepsilon}_{r \theta}, \theta\right), r=\dot{\varepsilon}_{r r}, \theta \theta-r \dot{\varepsilon}_{r r}, r+r\left(r \dot{\varepsilon}_{\theta \theta}\right), r r
$$

в полярной системе координат с полюсом в вершине трещины.

Определяющие соотношения (1), связывающие скорости деформаций ползучести и напряжения, замыкают сформулированную систему уравнений и в рамках предположения о реализации плоского напряженного состояния имеЮТ вид

$$
\begin{aligned}
& \dot{\varepsilon}_{r r}=(1 / 2) B\left(2 \sigma_{r r}-\sigma_{\theta \theta}\right) /\left(\sigma_{b}-\sigma_{e}\right), \quad \dot{\varepsilon}_{\theta \theta}=(1 / 2) B\left(2 \sigma_{\theta \theta}-\sigma_{r r}\right) /\left(\sigma_{b}-\sigma_{e}\right), \\
& \dot{\varepsilon}_{r \theta}=(3 / 2) B \sigma_{r \theta} /\left(\sigma_{b}-\sigma_{e}\right), \quad \sigma_{e}=\sqrt{\sigma_{r r}^{2}+\sigma_{\theta \theta}^{2}-\sigma_{r r} \sigma_{\theta \theta}+3 \sigma_{r \theta}^{2}} .
\end{aligned}
$$

Граничные условия задачи представляют собой условия отсутствия поверхностных усилий на берегах трещины

$$
\sigma_{\theta \theta}(r, \theta= \pm \pi)=0, \quad \sigma_{r \theta}(r, \theta= \pm \pi)=0 .
$$

Отметим, что в отличие от классических для механики трещин задач о трещине отрыва и сдвига, при изучении смешанного нагружения пластины с трещиной следует рассматривать всю плоскость $-\pi \leqslant \theta \leqslant \pi$ с разрезом, поскольку решение не обладает свойством симметрии или антисимметрии.

По мере удаления от вершины трещины уровень напряжений снижается и, следовательно, определяющие уравнения (4) постулируют линейную зависимость между скоростями деформаций ползучести и напряжениями. Поэтому граничные условия в бесконечно удаленной точке представляют собой условия асимптотического сближения искомого решения с решением задачи для линейно-вязкого материала:

$$
\begin{aligned}
\sigma_{r r} & =\frac{1}{\sqrt{2 \pi r}}\left\{C_{\mathrm{I}}[5 \cos (\theta / 2)-\cos (3 \theta / 2)]+C_{\text {П }}[-5 \sin (\theta / 2)+3 \sin (3 \theta / 2)]\right\}, \\
\sigma_{\theta \theta} & =\frac{1}{\sqrt{2 \pi r}}\left\{C_{\mathrm{I}}[3 \cos (\theta / 2)+\cos (3 \theta / 2)]+C_{\text {I }}[-3 \sin (\theta / 2)-3 \sin (3 \theta / 2)]\right\}, \\
\sigma_{r \theta} & =\frac{1}{\sqrt{2 \pi r}}\left\{C_{\mathrm{I}}[\sin (\theta / 2)+\sin (3 \theta / 2)]+C_{\text {П }}[\cos (\theta / 2)+3 \cos (3 \theta / 2)]\right\},
\end{aligned}
$$

где $C_{\mathrm{I}}, C_{\text {II }}-$ амплитудные коэффициенты, зависящие от геометрии образца и системы приложенных нагрузок.

Вид смешанного нагружения на удалении от кончика трещины может быть охарактеризован параметром $M^{i v}$, определяемым формулой

$$
M^{l v}=\frac{2}{\pi} \operatorname{arctg}\left|\lim _{r \rightarrow \infty} \frac{\sigma_{\theta \theta}(r, \theta=0)}{\sigma_{r \theta}(r, \theta=0)}\right|=\frac{2}{\pi} \operatorname{arctg}\left|\frac{C_{\text {I }}}{C_{\text {ㅍ }}}\right|,
$$


введенным по аналогии с упругим параметром смешанности нагружения [23, 25]. Введение безразмерных переменных согласно равенствам

$$
\hat{r}=r / L, \quad \hat{\sigma}_{i j}=\sigma_{i j} /\left(\sqrt{3} \sigma_{b} / 2\right), \quad \hat{\dot{\varepsilon}}_{i j}=\dot{\varepsilon}_{i j}(B / 2), \quad L=C^{*} /\left(B \sqrt{3} \sigma_{b} / 2\right)
$$

позволяет сформулировать систему уравнений в безразмерной форме. При этом уравнения равновесия (2) и условие совместности (3) сохраняют свою форму. Определяющие уравнения задачи после перехода к безразмерным величинам примут вид

$$
\begin{aligned}
& \hat{\dot{\varepsilon}}_{r r}=\left(2 \hat{\sigma}_{r r}-\hat{\sigma}_{\theta \theta}\right) /\left(1-\hat{\sigma}_{e}\right), \quad \hat{\dot{\varepsilon}}_{\theta \theta}=\left(2 \hat{\sigma}_{\theta \theta}-\hat{\sigma}_{r r}\right) /\left(1-\hat{\sigma}_{e}\right), \\
& \hat{\dot{\varepsilon}}_{r \theta}=3 \hat{\sigma}_{r \theta} /\left(1-\hat{\sigma}_{e}\right) .
\end{aligned}
$$

В дальнейшем для краткости знак^ опускается.

Асимптотический анализ. Для материала, следующего дробно-линейному закону ползучести (6)), можно искать поле напряжений вблизи устья трещины в виде разложения по собственным функциям

$$
\sigma_{i j}(r, \theta)=\sigma_{i j}^{(0)}(\theta)+r^{\alpha} \sigma_{i j}^{(1)}(\theta)+\ldots, \quad \sigma_{e}(r, \theta)=1-r^{\alpha} \sigma^{(1)}(\theta)+\ldots
$$

Тогда для определения функций $\sigma_{i j}^{(0)}(\theta)$ имеется система уравнений, следующая из двух уравнений равновесия (2) и условия предельного состояния $\sigma_{e}=1$ при $r=0$ :

$$
\begin{gathered}
\sigma_{r \theta, \theta}^{(0)}+\sigma_{r r}^{(0)}-\sigma_{\theta \theta}^{(0)}=0, \quad \sigma_{\theta \theta, \theta}^{(0)}+2 \sigma_{r \theta}^{(0)}=0 \\
\left(\sigma_{r r}^{(0)}\right)^{2}+\left(\sigma_{\theta \theta}^{(0)}\right)^{2}-\sigma_{r r}^{(0)} \sigma_{\theta \theta}^{(0)}+3\left(\sigma_{r \theta}^{(0)}\right)^{2}=1 .
\end{gathered}
$$

Для функций $\sigma_{i j}^{(0)}(\theta)$ формулируется, таким образом, краевая задача, аналогичная статически определимой задаче теории идеальной пластичности. Условию предельного состояния (8)) можно удовлетворить, если положить, что функции $\sigma_{i j}^{(0)}(\theta)$ задаются одной из двух альтернативных функциональных зависимостей

$$
\sigma_{r r}^{(0)}=\cos \left(\theta+c_{1}\right) / \sqrt{3}, \quad \sigma_{\theta \theta}^{(0)}=2 \cos \left(\theta+c_{1}\right) / \sqrt{3}, \quad \sigma_{r \theta}^{(0)}=\sin \left(\theta+c_{1}\right) / \sqrt{3},
$$

где $c_{1}$ - постоянная, определяемая из граничных условий задачи и условий непрерывности компонент тензора напряжений при переходе через лучи, разделяющие клинообразные области в окрестности вершины трещины, или

$$
\begin{aligned}
& \sigma_{r r}^{(0)}=a+b \cos 2 \vartheta(\theta)+c \sin 2 \vartheta(\theta), \sigma_{\theta \theta}^{(0)}=a-b \cos 2 \vartheta(\theta)-c \sin 2 \vartheta(\theta), \\
& \sigma_{r \theta}^{(0)}=-b \sin 2 \vartheta(\theta)+c \cos 2 \vartheta(\theta),
\end{aligned}
$$

где $a, b, c$ - такие постоянные, что $a^{2}+3 b^{2}+3 c^{2}=1$. Выполняя анализ, подобный проведенному для трещины смешанного типа в условиях плоского деформированного состояния [13], можно получить аналитическое решение системы уравнений (7), (8), удовлетворяющих граничным условиям на берегах трещины (5) для различных значений параметра смешанности нагружения $M^{p}$. Проведенный тщательный анализ структуры поля напряжений 
у вершины трещины позволяет установить, что поле напряжений для $M^{p}=$ $=0.25$ состоит из ансамбля семи секторов, для $M^{p}=0.5$ и $M^{p}=0.75$ поле напряжений состоит из пяти областей. Приведем точные формулы, задающие поле напряжений в окрестности вершины трещины смешанного типа в предположении реализации плоского напряженного состояния для характерных значений параметра $M^{p}$. Найденные поля напряжений показаны на рис. 1. На рис. 1, а приведено распределение напряжений, полученное для значения параметра смешанности нагружения $M^{p}=0$, что соответствует полю напряжений у вершины трещины чистого сдвига. На рис. $1, f$ показаны угловые распределения компонент тензора напряжений у вершины трещины отрыва, когда $M^{p}=1$.

Точками на графиках показаны угловые распределения компонент тензора напряжений у вершины трещины в материале со степенным определяющим законом в предельном случае, когда показатель степени (показатель упрочнения) стремится к бесконечности. Следует отметить, что условие наступления предельного состояния для дробно-линейного закона ползучести $\sigma_{e}=\sigma_{b}$ математически эквивалентно условию предельного состояния (условию идеальной пластичности) для степенного закона упрочнения (или степенного закона ползучести) при неограниченном увеличении показателя степени. В силу указанной аналогии между условием наступления предельного состояния и условием идеальной пластичности для верификации полученного аналитического решения является целесообразным сравнение найденного аналитического решения с численным распределением напряжений у вершины трещины для степенного определяющего закона для больших $n$ (в данном случае полагалось $n=300)$, что и показано на рис. 1 . Приведем итоговое распределение напряжений вблизи устья трещины для значения параметра смешанности нагружения $M^{p}=0.25$ (полученное поле напряжений показано на рис. $1, b)$ :

$$
\begin{aligned}
& \left\{\begin{array}{l}
-\pi \leqslant \theta \leqslant \theta_{\alpha}, \quad \theta_{\alpha} \approx-2.186257912=-125.26^{\circ}, \\
\sigma_{r r}^{(0)}=(1+\cos 2 \theta) / 2
\end{array}\right. \\
& \left\{\begin{array}{c}
\sigma_{r r}^{(0)} \\
\sigma_{\theta \theta}
\end{array}=(1+\cos 2 \theta) / 2,\right. \\
& \sigma_{\theta \theta}^{(0)}=(1-\cos 2 \theta) / 2 \text {, } \\
& \sigma_{r \theta}^{(0)}=-(1 / 2) \sin 2 \theta \text {; } \\
& \left\{\begin{array}{l}
\theta_{\alpha} \leqslant \theta \leqslant \theta_{\beta}, \quad \theta_{\beta} \approx-1.686144171=-96.60^{\circ}, \\
\sigma_{r r}^{(0)}=\cos \left(\theta+c_{3}\right) / \sqrt{3} \\
\sigma_{\theta \theta}^{(0)}=2 \cos \left(\theta+c_{3}\right) / \sqrt{3} \\
\sigma_{r \theta}^{(0)}=\sin \left(\theta+c_{3}\right) / \sqrt{3} \\
c_{3} \approx 1.230959418=70.53^{\circ}
\end{array}\right.
\end{aligned}
$$

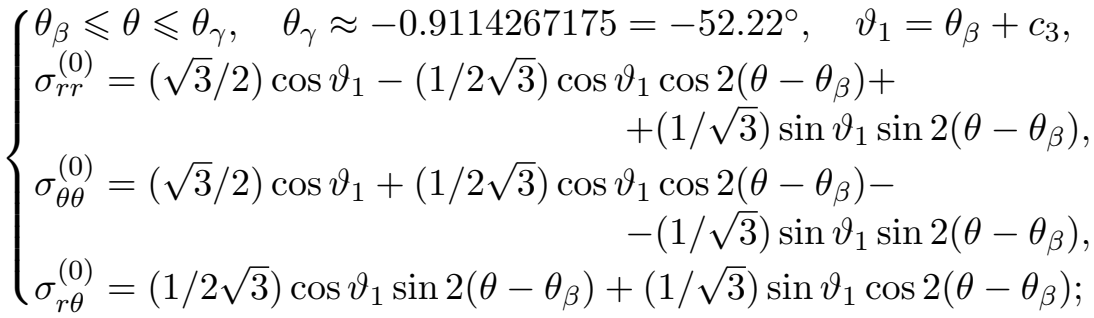



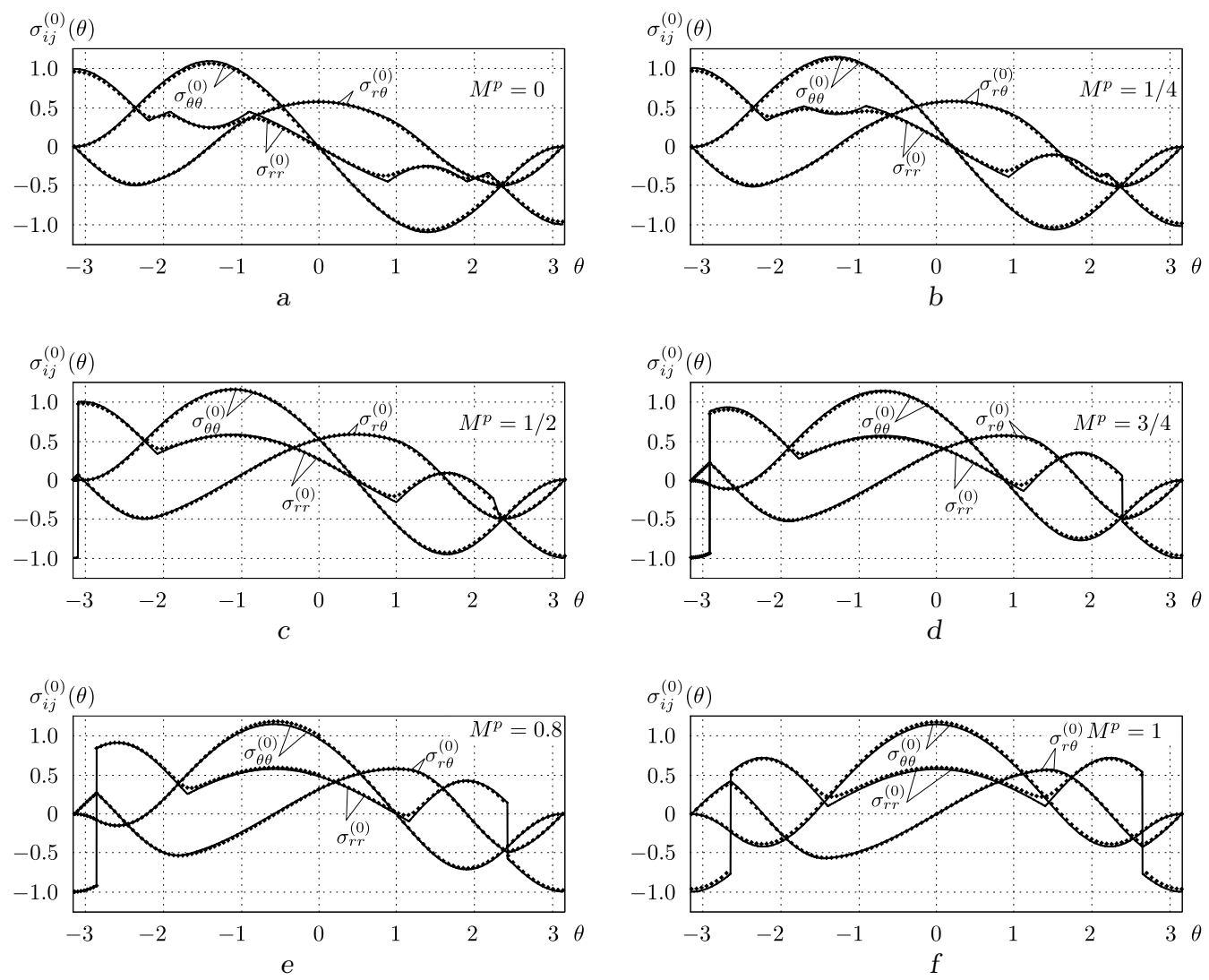

Рис. 1. Угловые распределения компонент тензора напряжений $\sigma_{i j}^{(0)}(\theta)$ у вершины трещины: сплошная линия - аналитическое решение; линия из маркеров - численный расчет для материала со степенным законом ползучести в предельном случае, когда $n=300$ и $M^{p}=0$ (a), $M^{p}=1 / 4(b), M^{p}=1 / 2(c), M^{p}=3 / 4(d), M^{p}=0.8(e), M^{p}=1(f)$

Figure 1. The angular distributions of the stress tensor components $\sigma_{i j}^{(0)}(\theta)$ at the crack tip. The solid line has been obtained using the analytical solution. The dotted line has been obtained using the numerical calculation for the power-law creeping material in the limit case when $n=300$ and $M^{p}=0$ (a), $M^{p}=1 / 4(b), M^{p}=1 / 2(c), M^{p}=3 / 4(d), M^{p}=0.8(e)$, and $M^{p}=1(f)$ 
$\left\{\begin{array}{l}\theta_{\gamma} \leqslant \theta \leqslant \theta_{\delta}, \quad \theta_{\delta} \approx 0.924219118=52.95^{\circ} \\ \sigma_{\theta \theta}^{(0)}=2 \sigma_{r r}^{(0)}=(2 / \sqrt{3}) \cos \left(\theta+c_{1}\right) \\ \sigma_{r \theta}^{(0)}=(1 / \sqrt{3}) \sin \left(\theta+c_{1}\right) \\ c_{1} \approx 1.366576756=78.30^{\circ}\end{array}\right.$

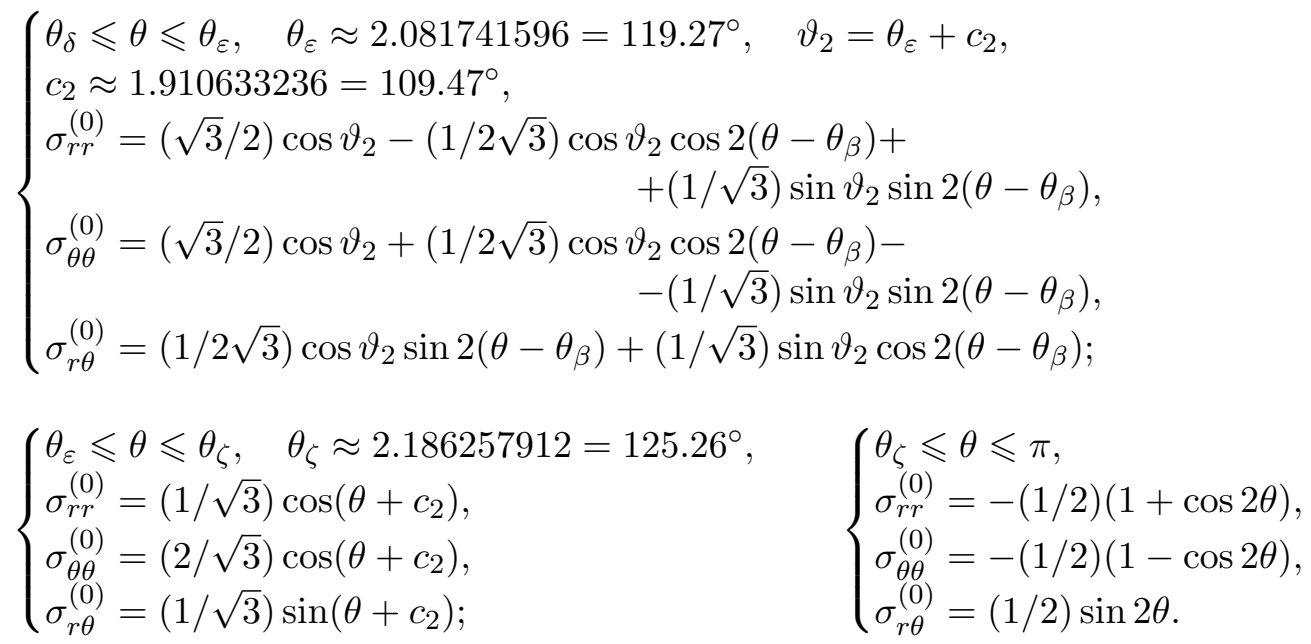

Приведем итоговое распределение напряжений $\sigma_{i j}^{(0)}(\theta)$ вблизи устья трещины для значения параметра смешанности нагружения $M^{p}=0.5$ (полученное поле напряжений показано на рис. $1, c)$ :

$$
\left\{\begin{array}{l}
-\pi \leqslant \theta \leqslant \theta_{\alpha}, \quad \theta_{\alpha} \approx-3.082081755=-176.60^{\circ}, \\
\sigma_{r r}^{(0)}=-(1 / 2)(1+\cos 2 \theta) \\
\sigma_{\theta \theta}^{(0)}=-(1 / 2)(1-\cos 2 \theta) \\
\sigma_{r \theta}^{(0)}=(1 / 2) \sin 2 \theta
\end{array}\right.
$$

$$
\begin{aligned}
& \left\{\begin{array}{l}
\theta_{\alpha} \leqslant \theta \leqslant \theta_{\beta}, \quad \theta_{\beta} \approx-2.069949494=-118.60^{\circ}, \\
\begin{array}{rl}
\sigma_{r r}^{(0)}=(\sqrt{3} / 2) \cos \vartheta_{1}-(1 /(2 \sqrt{3})) \cos \vartheta_{1} \cos 2\left(\theta-\theta_{\beta}\right)+ \\
\\
+(1 / \sqrt{3}) \sin \vartheta_{1} \sin 2\left(\theta-\theta_{\beta}\right),
\end{array}
\end{array}\right. \\
& \left\{\sigma_{\theta \theta}^{(0)}=(\sqrt{3} / 2) \cos \vartheta_{1}+(1 / 2 \sqrt{3}) \cos \vartheta_{1} \cos 2\left(\theta-\theta_{\beta}\right)-\right. \\
& -(1 / \sqrt{3}) \sin \vartheta_{1} \sin 2\left(\theta-\theta_{\beta}\right), \\
& \sigma_{r \theta}^{(0)}=(1 / 2 \sqrt{3}) \cos \vartheta_{1} \sin 2\left(\theta-\theta_{\beta}\right)+(1 / \sqrt{3}) \sin \vartheta_{1} \cos 2\left(\theta-\theta_{\beta}\right), \\
& \vartheta_{1}=\theta_{\beta}+c_{1}, \quad c_{1} \approx 1.107148718=63.43^{\circ} \text {; } \\
& \left\{\begin{array}{l}
\theta_{\beta} \leqslant \theta \leqslant \theta_{\gamma}, \quad \theta_{\gamma} \approx 0.988154515=56.62^{\circ}, \\
\sigma_{r r}^{(0)}=(1 / \sqrt{3}) \cos \left(\theta+c_{1}\right), \\
\sigma_{\theta \theta}^{(0)}=(2 / \sqrt{3}) \cos \left(\theta+c_{1}\right), \\
\sigma_{r \theta}^{(0)}=(1 / \sqrt{3}) \sin \left(\theta+c_{1}\right) ;
\end{array}\right.
\end{aligned}
$$




$$
\begin{aligned}
& \left\{\begin{array}{l}
\theta_{\gamma} \leqslant \theta \leqslant \theta_{\delta}, \quad \theta_{\delta} \approx 2.232478539=127.91^{\circ}, \\
\sigma_{r r}^{(0)}=a+(1 / 4)\left(1+\cos 2 \theta_{\delta}\right) \cos 2\left(\theta-\theta_{\delta}\right)+(1 / 2) \sin 2 \theta_{\delta} \sin 2\left(\theta-\theta_{\delta}\right), \\
\sigma_{\theta \theta}^{(0)}=a-(1 / 4)\left(1+\cos 2 \theta_{\delta}\right) \cos 2\left(\theta-\theta_{\delta}\right)-(1 / 2) \sin 2 \theta_{\delta} \sin 2\left(\theta-\theta_{\delta}\right), \\
\sigma_{r \theta}^{(0)}=-(1 / 4)\left(1+\cos 2 \theta_{\delta}\right) \sin 2\left(\theta-\theta_{\delta}\right)+(1 / 2) \sin 2 \theta_{\delta} \cos 2\left(\theta-\theta_{\delta}\right), \\
a=\left(-1+3 \cos 2 \theta_{\delta}\right) / 4 ;
\end{array}\right. \\
& \left\{\begin{array}{l}
\theta_{\delta} \leqslant \theta \leqslant \pi, \\
\sigma_{r r}^{(0)}=-(1 / 2)(1+\cos 2 \theta), \\
\sigma_{\theta \theta}^{(0)}=-(1 / 2)(1-\cos 2 \theta), \\
\sigma_{r \theta}^{(0)}=(1 / 2) \sin 2 \theta .
\end{array}\right.
\end{aligned}
$$

Угловое распределение $\sigma_{i j}^{(0)}(\theta)$ компонент тензора напряжений вблизи устья трещины для значения параметра смешанности нагружения $M^{p}=0.75$ имеет вид

$$
\begin{aligned}
& \left\{\begin{array}{l}
-\pi \leqslant \theta \leqslant \theta_{\alpha}, \quad \theta_{\alpha} \approx-2.904522467=-166.42^{\circ}, \\
\sigma_{r r}^{(0)}=-(1 / 2)(1+\cos 2 \theta), \\
\sigma_{\theta \theta}^{(0)}=-(1 / 2)(1-\cos 2 \theta), \\
\sigma_{r \theta}^{(0)}=(1 / 2) \sin 2 \theta ;
\end{array}\right. \\
& \left\{\begin{array}{l}
\theta_{\alpha} \leqslant \theta \leqslant \theta_{\beta}, \quad \theta_{\beta} \approx-1.759907174=-100.84^{\circ}, \\
\sigma_{r r}^{(0)}=(\sqrt{3} / 2) \cos \vartheta_{1}-(1 / 2 \sqrt{3}) \cos \vartheta_{1} \cos 2\left(\theta-\theta_{\beta}\right)+ \\
\sigma_{\theta \theta}^{(0)}=(\sqrt{3} / 2) \cos \vartheta_{1}+(1 / 2 \sqrt{3}) \cos \vartheta_{1} \cos 2\left(\theta-\theta_{\beta}\right)- \\
\sigma_{r \theta}^{(0)}=(1 / 2 \sqrt{3}) \cos \vartheta_{1} \sin 2\left(\theta-\theta_{\beta}\right)+(1 / \sqrt{3}) \sin 2\left(\theta-\vartheta_{1} \sin 2\left(\theta-\theta_{\beta}\right),\right. \\
\vartheta_{1}=\vartheta_{\beta}+c_{1}, \quad c_{1} \approx 0.6918358137=39.64^{\circ} ;
\end{array}\right. \\
& \left\{\begin{array}{l}
\theta_{\beta} \leqslant \theta \leqslant \theta_{\gamma}, \quad \theta_{\gamma} \approx 1.119349842=64.13^{\circ}, \\
\sigma_{r r}^{(0)}=(1 / \sqrt{3}) \cos \left(\theta+c_{1}\right), \\
\sigma_{\theta \theta}^{(0)}=(2 / \sqrt{3}) \cos \left(\theta+c_{1}\right), \\
\sigma_{r \theta}^{(0)}=(1 / \sqrt{3}) \sin \left(\theta+c_{1}\right) ;
\end{array}\right. \\
& \left\{\begin{array}{l}
\theta_{\gamma} \leqslant \theta \leqslant \theta_{\delta}, \quad \theta_{\delta} \approx 2.385429690=136.68^{\circ}, \\
\sigma_{r r}^{(0)}=a+(1 / 4)\left(1+\cos 2 \theta_{\delta}\right) \cos 2\left(\theta-\theta_{\delta}\right)+(1 / 2) \sin 2 \theta_{\delta} \sin 2\left(\theta-\theta_{\delta}\right), \\
\sigma_{\theta \theta}^{(0)}=a-(1 / 4)\left(1+\cos 2 \theta_{\delta}\right) \cos 2\left(\theta-\theta_{\delta}\right)-(1 / 2) \sin 2 \theta_{\delta} \sin 2\left(\theta-\theta_{\delta}\right), \\
\sigma_{r \theta}^{(0)}=-(1 / 4)\left(1+\cos 2 \theta_{\delta}\right) \sin 2\left(\theta-\theta_{\delta}\right)+(1 / 2) \sin 2 \theta_{\delta} \cos 2\left(\theta-\theta_{\delta}\right), \\
\sigma_{r \theta}^{(0)}=(1 / 2) \sin 2 \theta .
\end{array}\right.
\end{aligned}
$$

Полученное поле напряжений показано на рис. $1, d$. 


\section{Анализ поля скоростей деформаций ползучести у вершины трещины.} Определяющие соотношения и найденное поле напряжений вблизи кончика трещины позволяют вывести асимптотические формулы для поля скоростей деформаций ползучести. Ввиду равенств (6) и (9) итоговое распределение скоростей деформаций у вершины трещины для $M^{p}=0.25$ имеет вид

$$
\begin{aligned}
& \left\{\begin{array} { l } 
{ - \pi \leqslant \theta < \theta _ { \alpha } , \quad \theta _ { \alpha } \approx - 1 2 5 . 2 6 ^ { \circ } , } \\
{ \dot { \varepsilon } _ { r r } = \frac { 1 } { 2 } \frac { 1 + 3 \operatorname { c o s } 2 \theta } { r ^ { \alpha } \sigma ^ { ( 1 ) } } , } \\
{ \dot { \varepsilon } _ { \theta \theta } = \frac { 1 } { 2 } \frac { 1 - 3 \operatorname { c o s } 2 \theta } { r ^ { \alpha } \sigma ^ { ( 1 ) } } , } \\
{ \dot { \varepsilon } _ { r \theta } = - \frac { 3 } { 2 } \frac { \operatorname { s i n } 2 \theta } { r ^ { \alpha } \sigma ^ { ( 1 ) } } ; }
\end{array} \quad \left\{\begin{array}{l}
\theta_{\alpha}<\theta<\theta_{\beta}, \quad \theta_{\beta} \approx-96.60^{\circ}, \\
\dot{\varepsilon}_{r r}=0, \\
\dot{\varepsilon}_{\theta \theta}=\sqrt{3} \frac{\cos \left(\theta+c_{3}\right)}{r^{\alpha} \sigma^{(2)}}, \\
\dot{\varepsilon}_{r \theta}=\sqrt{3} \frac{\sin \left(\theta+c_{3}\right)}{r^{\alpha} \sigma^{(2)}} ;
\end{array}\right.\right. \\
& \left(\theta_{\beta}<\theta<\theta_{\gamma}, \quad \theta_{\gamma} \approx-52.22^{\circ},\right. \\
& \left\{\begin{array}{l}
\dot{\varepsilon}_{r r}=\frac{\sqrt{3}}{2} \frac{\cos \vartheta_{1}-\cos \vartheta_{1} \cos 2\left(\theta-\theta_{\beta}\right)+2 \sin \vartheta_{1} \sin 2\left(\theta-\theta_{\beta}\right)}{r^{\alpha} \sigma^{(3)}}, \\
\dot{\varepsilon}_{\theta \theta}=\frac{\sqrt{3}}{2} \frac{\cos \vartheta_{1}+\cos \vartheta_{1} \cos 2\left(\theta-\theta_{\beta}\right)-2 \sin \vartheta_{1} \sin 2\left(\theta-\theta_{\beta}\right)}{r^{\alpha} \sigma^{(3)}},
\end{array}\right. \\
& \dot{\varepsilon}_{r \theta}=\frac{\sqrt{3}}{2} \frac{\cos \vartheta_{1} \sin 2\left(\theta-\theta_{\beta}\right)+2 \sin \vartheta_{1} \cos 2\left(\theta-\theta_{\beta}\right)}{r^{\alpha} \sigma^{(3)}} ; \\
& \left\{\begin{array}{l}
\theta_{\gamma}<\theta<\theta_{\delta}, \quad \theta_{\delta} \approx 52.95^{\circ} \\
\dot{\varepsilon}_{r r}=0
\end{array}\right. \\
& \left\{\dot{\varepsilon}_{\theta \theta}=\sqrt{3} \frac{\cos \left(\theta+c_{1}\right)}{r^{\alpha} \sigma^{(4)}},\right. \\
& \dot{\varepsilon}_{r \theta}=\sqrt{3} \frac{\sin \left(\theta+c_{1}\right)}{r^{\alpha} \sigma^{(4)}} ; \\
& \left(\theta_{\delta}<\theta<\theta_{\varepsilon}, \quad \theta_{\varepsilon} \approx 119.27^{\circ},\right. \\
& \begin{array}{l}
\dot{\varepsilon}_{r r}=\frac{\sqrt{3}}{2} \frac{\cos \vartheta_{2}-\cos \vartheta_{2} \cos 2\left(\theta-\theta_{\beta}\right)+2 \sin \vartheta_{2} \sin 2\left(\theta-\theta_{\beta}\right)}{r^{\alpha} \sigma^{(5)}} \\
\dot{\varepsilon}_{\theta \theta}=\frac{\sqrt{3}}{2} \frac{\cos \vartheta_{2}+\cos \vartheta_{2} \cos 2\left(\theta-\theta_{\beta}\right)-2 \sin \vartheta_{2} \sin 2\left(\theta-\theta_{\beta}\right)}{r^{\alpha} \sigma^{(5)}}
\end{array} \\
& \dot{\varepsilon}_{r \theta}=\frac{\sqrt{3}}{2} \frac{\cos \vartheta_{2} \sin 2\left(\theta-\theta_{\beta}\right)+2 \sin \vartheta_{2} \cos 2\left(\theta-\theta_{\beta}\right)}{r^{\alpha} \sigma^{(5)}} ;
\end{aligned}
$$$$
\left\{\begin{array} { l } 
{ \theta _ { \varepsilon } < \theta < \theta _ { \zeta } , \quad \theta _ { \zeta } \approx 1 2 5 . 2 6 ^ { \circ } , } \\
{ \dot { \varepsilon } _ { r r } = 0 , } \\
{ \dot { \varepsilon } _ { \theta \theta } = \sqrt { 3 } \frac { \operatorname { c o s } ( \theta + c _ { 2 } ) } { r ^ { \alpha } \sigma ^ { ( 6 ) } } , } \\
{ \dot { \varepsilon } _ { r \theta } = \sqrt { 3 } \frac { \operatorname { s i n } ( \theta + c _ { 2 } ) } { r ^ { \alpha } \sigma ^ { ( 6 ) } } ; }
\end{array} \quad \left\{\begin{array}{l}
\theta_{\zeta}<\theta \leqslant \pi \\
\dot{\varepsilon}_{r r}=-\frac{1}{2} \frac{1+3 \cos 2 \theta}{r^{\alpha} \sigma^{(7)}} \\
\dot{\varepsilon}_{\theta \theta}=-\frac{1}{2} \frac{1-3 \cos 2 \theta}{r^{\alpha} \sigma^{(7)}} \\
\dot{\varepsilon}_{r \theta}=\frac{3}{2} \frac{\sin 2 \theta}{r^{\alpha} \sigma^{(7)}}
\end{array}\right.\right.
$$

Для следующего характерного значения параметра смешанности нагружения $M^{p}=0.5$ асимптотическое представление поля скоростей деформаций 
ползучести определяется формулами

$$
\begin{aligned}
& \left(-\pi \leqslant \theta<\theta_{\alpha}, \quad \theta_{\alpha} \approx-176.60^{\circ}\right. \text {, } \\
& \dot{\varepsilon}_{r r}=-\frac{1}{2} \frac{1+3 \cos 2 \theta}{r^{\alpha} \sigma^{(1)}}, \\
& \dot{\varepsilon}_{\theta \theta}=-\frac{1}{2} \frac{1-3 \cos 2 \theta}{r^{\alpha} \sigma^{(1)}}, \\
& \dot{\varepsilon}_{r \theta}=\frac{3}{2} \frac{\sin 2 \theta}{r^{\alpha} \sigma^{(1)}} \\
& \left(\theta_{\alpha}<\theta<\theta_{\beta}, \quad \theta_{\beta} \approx-118.60^{\circ},\right. \\
& \dot{\varepsilon}_{r r}=\frac{\sqrt{3}}{2} \frac{\cos \vartheta_{1}-\cos \vartheta_{1} \cos 2\left(\theta-\theta_{\beta}\right)+2 \sin \vartheta_{1} \sin 2\left(\theta-\theta_{\beta}\right)}{r^{\alpha} \sigma^{(2)}}, \\
& \left\{\dot{\varepsilon}_{\theta \theta}=\frac{\sqrt{3}}{2} \frac{\cos \vartheta_{1}+\cos \vartheta_{1} \cos 2\left(\theta-\theta_{\beta}\right)-2 \sin \vartheta_{1} \sin 2\left(\theta-\theta_{\beta}\right)}{r^{\alpha} \sigma^{(2)}},\right. \\
& \dot{\varepsilon}_{r \theta}=\frac{\sqrt{3}}{2} \frac{\cos \vartheta_{1} \sin 2\left(\theta-\theta_{\beta}\right)+2 \sin \vartheta_{1} \cos 2\left(\theta-\theta_{\beta}\right)}{r^{\alpha} \sigma^{(2)}} ; \\
& \left\{\begin{array}{l}
\theta_{\beta}<\theta<\theta_{\gamma}, \quad \theta_{\gamma} \approx 56.62^{\circ}, \\
\dot{\varepsilon}_{r r}=0, \\
\dot{\varepsilon}_{\theta \theta}=\sqrt{3} \frac{\cos \left(\theta+c_{1}\right)}{r^{\alpha} \sigma^{(3)}}, \\
\dot{\varepsilon}_{r \theta}=\sqrt{3} \frac{\sin \left(\theta+c_{1}\right)}{r^{\alpha} \sigma^{(3)}} ;
\end{array}\right. \\
& \left\{\begin{array}{l}
\theta_{\gamma}<\theta<\theta_{\delta}, \quad \theta_{\delta} \approx 127.91^{\circ}, \\
\dot{\varepsilon}_{r r}=\frac{1}{r^{\alpha} \sigma^{(4)}}\left\{\left(-1+3 \cos 2 \theta_{\delta}\right) / 4+(3 / 4)\left(1+\cos 2 \theta_{\delta}\right) \cos 2\left(\theta-\theta_{\delta}\right)+\right.
\end{array}\right. \\
& \left.+(3 / 2) \sin 2 \theta_{\delta} \sin 2\left(\theta-\theta_{\delta}\right)\right\} \\
& \dot{\varepsilon}_{\theta \theta}=\frac{1}{r^{\alpha} \sigma^{(4)}}\left\{\left(-1+3 \cos 2 \theta_{\delta}\right) / 4-(3 / 4)\left(1+\cos 2 \theta_{\delta}\right) \cos 2\left(\theta-\theta_{\delta}\right)-\right. \\
& \left.-(3 / 2) \sin 2 \theta_{\delta} \sin 2\left(\theta-\theta_{\delta}\right)\right\}, \\
& \dot{\varepsilon}_{r \theta}=\frac{-(3 / 4)\left(1+\cos 2 \theta_{\delta}\right) \sin 2\left(\theta-\theta_{\delta}\right)+(3 / 2) \sin 2 \theta_{\delta} \cos 2\left(\theta-\theta_{\delta}\right)}{r^{\alpha} \sigma^{(4)}} ; \\
& \left\{\begin{array}{l}
\theta_{\delta}<\theta \leqslant \pi, \\
\dot{\varepsilon}_{r r}=-\frac{1}{2} \frac{1+3 \cos 2 \theta}{r^{\alpha} \sigma^{(5)}}, \\
\dot{\varepsilon}_{\theta \theta}=-\frac{1}{2} \frac{1-3 \cos 2 \theta}{r^{\alpha} \sigma^{(5)}}, \\
\dot{\varepsilon}_{r \theta}=\frac{3}{2} \frac{\sin 2 \theta}{r^{\alpha} \sigma^{(5)}} .
\end{array}\right.
\end{aligned}
$$

Структура поля скоростей деформаций для $M^{p}=0.75$ совпадает с совокупностью формул (11), но углы, соответствующие линиям раздела секторов, имеют другие числовые значения, указанные в формулах (10). На рис. 2 показаны угловые распределения скоростей деформаций ползучести в материале 

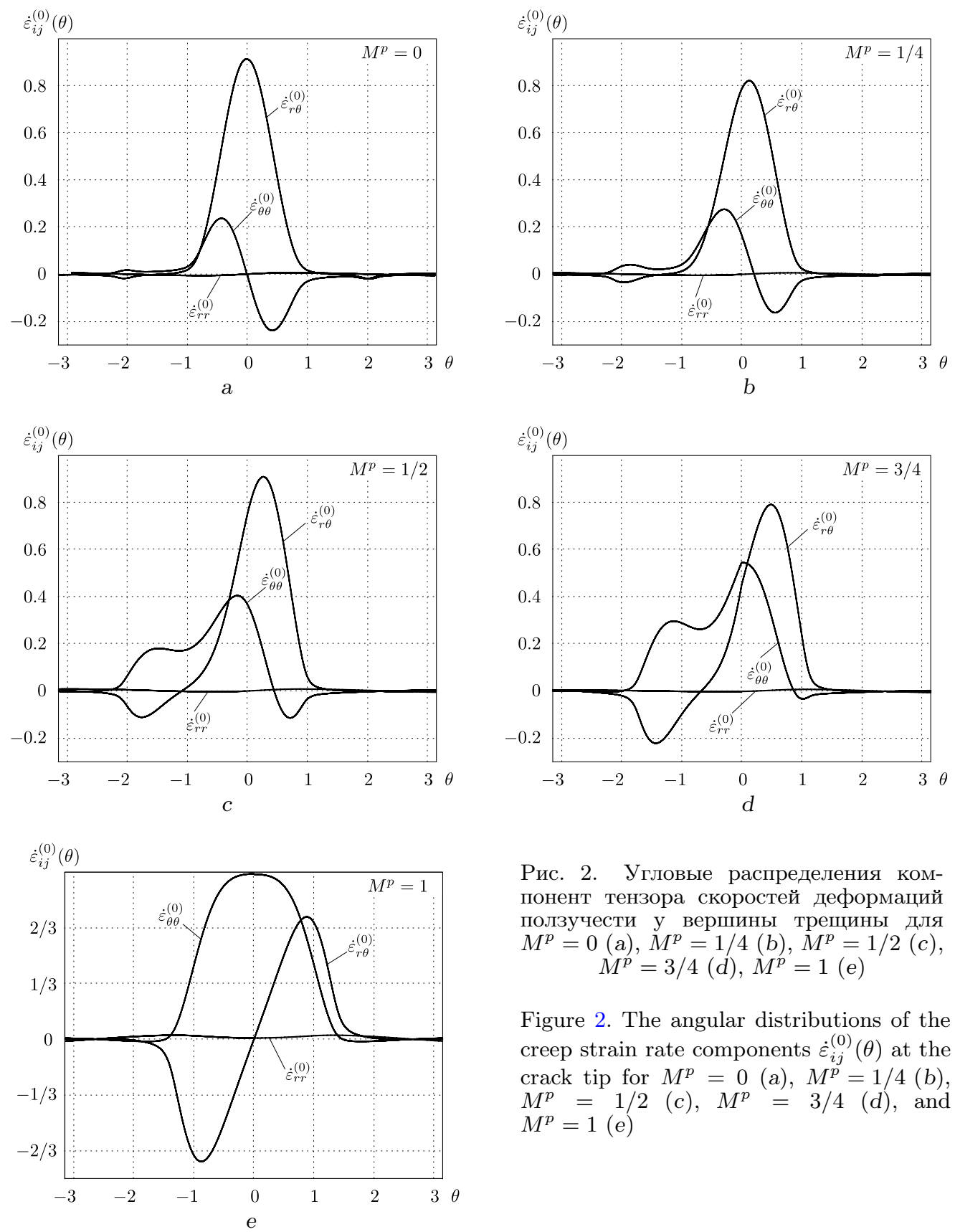

Рис. 2. Угловые распределения компонент тензора скоростей деформаций ползучести у вершины трещины для $M^{p}=0(\mathrm{a}), M^{p}=1 / 4(b), M^{p}=1 / 2(c)$, $M^{p}=3 / 4(d), M^{p}=1(e)$

Figure 2. The angular distributions of the creep strain rate components $\dot{\varepsilon}_{i j}^{(0)}(\theta)$ at the crack tip for $M^{p}=0(\mathrm{a}), M^{p}=1 / 4(b)$, $M^{p}=1 / 2(c), M^{p}=3 / 4(d)$, and $M^{p}=1(e)$ 
со степенным законом ползучести в предельном случае при неограниченном возрастании показателя степени. В ходе численного счета полагалось, что $n=300$. На рисунках ясно видно, что имеются интервалы изменения полярного угла $\theta$, внутри которых коэффициенты асимптотических разложений скоростей деформаций ползучести равны нулю, что свидетельствует о более слабой особенности поля скоростей деформаций по сравнению с $r^{-1}$. Например, на рис. $2, b$ - это области $-\pi<\theta<\theta_{\alpha}, \theta_{\beta}<\theta<\theta_{\gamma}$ и $\theta_{\delta}<\theta<\pi$. На остальных интервалах изменения полярного угла $\theta$ скорости деформаций ползучести имеют особенность вида $r^{-1}$.

Выводы. В работе найдены аналитические решения задачи определения напряженно-деформированного состояния в непосредственной окрестности вершины трещины в образце, находящемся в условиях смешанного нагружения для различных значений коэффициента смешанности нагружения, определяющего вид нагружения. Показано, что поле напряжений состоит из различных областей (секторов), внутри которых компоненты тензора напряжений определяются различными функциональными зависимостями. Границы введенных секторов находятся численно из решения системы трансцендентных уравнений. Приведено сравнение приближенного аналитического решения с численным решением задачи для материала, следующего степенному закону Бейли-Нортона теории установившейся ползучести в предельном случае, когда показатель нелинейности материала неограниченно возрастает. Для сравнения построены угловые распределения компонент тензоров напряжений и скоростей деформаций ползучести в материале со степенным законом для различных значений показателя нелинейности материала. Аналитическое и численное решения совпадают, что подтверждает достоверность результатов. Полученное решение позволяет пролить свет на поле деформаций у устья трещины в идеально пластическом материале, поскольку условие наступления предельного состояния аналогично условию наступления пластического течения Мизеса. Построенные графики распределений компонент тензора напряжений ясно показывают, что радиальное напряжение $\sigma_{r r}^{(0)}(\theta)$ вблизи $\theta=-\pi$ для $M^{p} \geqslant 0.4$ претерпевает резкие изменения, которые следует учитывать при построении численного решения соответствующей задачи для степенного закона теории ползучести в условиях смешанного нагружения.

Работа выполнена при поддержке РФФИ (проект № 13-01-97009-р).

This work is supported by RFBR, project no. 13-01-97009-p.

\section{СПИСОК ЛИТЕРАТУРЫ/ REFERENCES}

1. M. Kuna, Finite Elements in Fracture Mechanics, Theory-Numerics-Applications, Solid Mechanics and Its Applications, vol. 201, Dordrecht, Springer, 2013, xxv+464 pp. doi : 10 . 1007/978-94-007-6680-8.

2. Л. В. Степанова, Математические методь механики разрушения, М.: Физматлит, 2009. 336 с. [L. V. Stepanova, Matematicheskiye metody mekhaniki razrusheniya [Mathematical methods of fracture mechanics], Moscow, Fizmatlit, 2009, 336 pp. (In Russian)]

3. J. Pan, C. Lin, "Analytical solutions for crack-tip sectors in perfectly plastic Mises materials under mixed in-plane and out-of-plane shear loading conditions", Eng. Frac. Mech., 2006, vol. 73, no. 13, pp. 1797-1813. doi: 10.1016/j.engfracmech.2006.03.002.

4. M. Rahman, J. W. Hancock, "Elastic perfectly-plastic asymptotic mixed mode crack tip 
fields in plane stress", Int. J. Sol. Struct., 2006, vol.43, no. 13, pp. 3692-3704. doi: j. ijsolstr.2005.05.051.

5. V. N. Shlyannikov, B. V. Ilchenko, N. V. Boychenko, "Biaxial Loading Effect on HigherOrder Crack Tip Parameters", J. ASTM International, 2008, vol. 5, no. 8, JAI101548. doi : $10.1520 /$ JAI101548.

6. В. Н. Шлянников, С. Ю. Кислова, "Параметры смешанных форм деформирования для трещины в виде математического разреза" // Изв. Сарат. ун-та. Нов. сер. Сер. Математика. Механика. Информатика, 2009. Т. 9, №1. C. 77-84. [V. N. Shlyannikov, S. Yu. Kislova, "Mode mixity parameters for mathematical crack type", Izv. Saratov. Univ. Mat. Mekh. Inform., 2009, vol. 9, no. 1, pp. 77-84. (In Russian)].

7. В. Н. Шлянников, А. В. Туманов, "Упругие параметры смешанных форм деформирования полуэллиптической трещины при двухосном нагружении" // Изв. Cарат. унта. Нов. сер. Сер. Математика. Механика. Информатика, 2010. Т. 10, № 2. С. 73-80. [V. N. Shlyannikov, A. V. Tumanov, "Elastic mode mixity parameters for semi-elliptical crack under biaxial loading", Izv. Saratov. Univ. Mat. Mekh. Inform., 2010, vol. 10, no. 2, pp. 73-80. (In Russian)].

8. В. Н. Шлянников, "Решение задач нелинейного деформирования и разрушения материалов при сложном напряженном состоянии" // Физ. мезомех., 2012. №1. С. 57-67; V. N. Shlyannikov, "Solution of nonlinear strain and fracture problems for materials in complex stress states", Phys. Mesomech., 2012, vol. 15, no. 3-4, pp. 176-184. doi: 10.1134/ S102995991202004X.

9. С. Ю. Кислова, В. Н. Шлянников, "Упругие и пластические параметры смешанности экспериментальных образцов различной геометрии" // Труды Академэнерго, 2012. № 1. C. 101-112. [S. Yu. Kislova, V. N. Shlyannikov, "Elastic and plastic mode mixity parameters for test specimens with different geometries", Trudy Akademenergo, 2012, no. 1, pp. 101-112. (In Russian)].

10. К. А. Вансович, В. И. Ядров, "Усталостные испытания стальных крестообразных образцов с поверхностной трещиной при двухосном нагружении" // Омский научный вестник, 2012. №3(113). C. 117-121. [K. A. Vansovich, V. I. Yadrov, "Fatigue tests of steel cruciform specimens with the surface crack at biaxial loading", Omskiy nauchnyy vestnik, 2012, no. 3(113), pp. 117-121. (In Russian)].

11. К. А. Вансович, В. И. Ядров, "Экспериментальное изучение скорости роста поверхностных трещин в алюминиевом сплаве AK6 и в стали 20 при двухосном нагружении" // Известия Самарского научного центра Российской академии наук, 2012. Т. 15, № 4(2). C. 436-438. [K. A. Vansovich, V. I. Yadrov, "Experimental research the growth rate of surface cracks in AK6 aluminium alloy and St. 20 steel at biaxial loading", Izvestiya Samarskogo nauchnogo tsentra Rossiyskoy akademii nauk, 2012, vol.15, no.4(2), pp. 436438. (In Russian)].

12. В. Н. Шлянников, А. П. Захаров, А. А. Герасименко, "Характеристики циклической трещиностойкости стали СТ-3 при двухосном нагружении" // Труды Академэнерго, 2013. № 4. C. 91-101. [V. N. Shlyannikov, A. P. Zakharov, A. A. Gerasimenko, "Fatigue crack growth for steel St-3 under biaxial loading", Trudy Akademenergo, 2013, no. 4, pp. 91101. (In Russian)].

13. Л. В. Степанова, Т. Б. Элекина, "Смешанное нагружение (нормальный отрыв и поперечный сдвиг) элемента конструкции с трещиной в материале с дробно-линейным законом ползучести" // Вестник Самарского государственного университета, 2009. № 2(68). C. 123-139. [L. V. Stepanova, T. B. Elekina, "Combined mode loading (normal tracture model and in-plane shear) of the element of construction with in a material with the linear-fractional creep principle", Vestnik Samarskogo Gosudarstvennogo Universiteta, 2009, no. 2(68), pp. 123-139. (In Russian)].

14. Т. Е. Герасимова, П. Н. Ломаков, Л. В. Степанова, "Цифровая фотомеханика: численная обработка результатов оптоэлектронных измерений и ее приложение к задачам механики разрушения" // Вестник Самарского государственного университета, 2013. № 9/2(110). C. 64-74. [T. E. Gerasimova, P. N. Lomakov, L. V. Stepanova, "Numerical 
photomechanics. Numerical processing of photoelasticity experiments and its application to the problems of fracture mechanics problems", Vestnik Samarskogo Gosudarstvennogo Universiteta, 2013, no. 9/2(110), pp. 64-74. (In Russian)].

15. Л. В. Степанова, Е. М. Адылина, “Автомодельное решение задачи о смешанном деформировании пластины с трещиной в среде с поврежденностью" // Вестник Самарского государственного университета, 2013. №9/1(110). C. 76-93. [L. V. Stepanova, E. M. Adylina, "Self-similar solution to the problem of mixed deformation of plate with a crack tip in a damaged medium", Vestnik Samarskogo Gosudarstvennogo Universiteta, 2013, no. 9/1(110), pp. 76-93. (In Russian)].

16. Л. В. Степанова, "О собственных значениях в задаче о трещине антиплоского сдвига в материале со степенными определяющими уравнениями" // ПМТФ, 2008. Т. 49, № 1 . C. 173-180; L. V. Stepanova, "Eigenvalues of the antiplane-shear crack problem for a powerlaw material", J. Appl. Mech. Tech. Phys., 2008, vol.49, no.1, pp. 142-147. doi: 10.1007/ s10808-008-0021-7.

17. Л. В. Степанова, М. Е. Федина, "Автомодельное решение задачи о трещине антиплоского сдвига в связанной постановке (связка «ползучесть - поврежденность»)" // Вестник Самарского государственного университета, 2000. №4(18). С. 128-145. [L. V. Stepanova, M. E. Fedina, "Self-similar solution of the problem of an antiplane shear crack in a coupled formulation ('creep-damage' coupling)", Vestnik Samarskogo Gosudarstvennogo Universiteta, 2000, no.4(18), pp. 128-145. (In Russian)].

18. Л. В. Степанова, М. Е. Федина, "О геометрии области полностью поврежденного материала у вершины трещины антиплоского сдвига в связанной постановке задачи (связка «ползучесть - поврежденность»)" // Вестник Самарского государственного университета, 2001. №2(20). C. 87-113. [L. V. Stepanova, M. E. Fedina, "On the geometry of the completely damaged material region at the antiplane-shear crack tip in the conjugate formulation of the problem ('creep-damage' coupling)", Vestnik Samarskogo Gosudarstvennogo Universiteta, 2001, no. 2(20), pp. 87-113. (In Russian)].

19. Л.В.Степанова, М. Е. Федина, “Автомодельное решение задачи о трещине антиплоского сдвига в связанной постановке (ползучесть-поврежденность)" // ПМТФ, 2002. Т. 43, № 5. C. 114-123; L. V. Stepanova, M. E. Fedina, "Self-Similar Solution of the Antiplane Shear Fracture Problem in a Coupled Formulation (Creep-Damage)", J. Appl. Mech. Tech. Phys., 2002, vol. 43, no. 5, pp. 731-738. doi: 10.1023/A:1019848121449.

20. Е. В. Ломакин, А. М. Мельников, "Пластическое плоское напряженное состояние тел, свойства которых зависят от вида напряженного состояния" // Вычислительная механика сплошных сред, 2009. Т. 2, № 2. С. 48-64 doi: 10.7242/1999-6691/2009.2.2.12. [E. V. Lomakin, M. A. Melnikov, "Plane stress state of media with plastic properties sensitive to the type of stress", Computational Continuum Mechanics, 2009, vol. 2, no. 2, pp. 48-64. (In Russian)].

21. А. М. Мельников, "Плоское напряженное состояние полосы из материала, свойства которого зависят от вида напряженного состояния" // Вестник Нижегородского университета им. Н. И. Лобачевского, 2011. № 4(5). С. 2352-2353. [A. M. Melnikov, "Plane stress of a stripe made of a stress-state-dependent material with angular cutouts", Vestnik Nizhegorodskogo Universiteta im. N. I. Lobachevskogo, 2011, no.4(5), pp. 2352-2353. (In Russian)].

22. Е. В. Ломакин, А. М. Мельников, “Задачи плоского напряженного состояния тел с вырезами, пластические свойства которых зависят от вида напряженого состояния" // Известия Российской академии наук. Механика твердого тела, 2011. №1. С. 77-94; E. V. Lomakin, A. M. Mel'nikov, "Plane stress state problems for notched bodies whose plastic properties depend on the form of the stress state", Mechanics of Solids, 2011, vol. 46, no. 1, pp. 62-69. doi: 10.3103/S0025654411010092.

23. C. F. Shih, Elastic-plastic analysis of combined mode crack problems, Ph. D. Thesis, Cambridge, M.A., Harvard University, 1973.

24. C. F. Shih, J. W. Hutchinson, "Fully Plastic Solutions and Large Scale Yielding Estimates 
for Plane Stress Crack Problems", J. Eng. Mater. Technol., 1976, vol. 98, no. 4, pp. 289-295. doi : 10.1115/1.3443380.

25. C. F. Shih, "Small-Scale Yielding Analysis of Mixed Mode Plane-Strain Crack Problems", Fracture Analysis (STP560), Proceedings of the 1973 National Symposium on Fracture Mechanics, Part II, 1974, pp. 187-210. doi : 10.1520/STP33141S doi: 10.1520/STP560-EB.

26. V. N. Shlyannikov, Elastic-Plastic Mixed-Mode Fracture Criteria and Parameters, Lecture Notes in Applied Mechanics, vol.7, Berlin, Springer, 2003, viii+246 pp. doi: 10.1007/ 978-3-540-45836-4.

27. Mixed-Mode Crack Behavoir (STP1359), eds. K. J. Miller, D. L. McDowell, 1999, ix+337 pp. doi : 10.1520/stp1359-eb.

Поступила в редакцию 11/XII/2013;

в окончательном варианте - 17/II/2014;

принята в печать $-21 / \mathrm{II} / 2014$.

MSC: 74R20; 74G70, 74C05

\section{STRESS FIELD NEAR THE MIXED MODE CRACK TIP UNDER PLANE STRESS CONDITIONS}

\section{V. Stepanova, E. M. Adylina}

Samara State University,

1, Academician Pavlov st., Samara, 443011, Russian Federation.

The asymptotical solution to the problem of the mixed loading of the cracked specimen under plane stress conditions in materials with fractional-linear constitutive relations of steady-state creep is given. The stresses and creep strain rates in the vicinity of the mixed mode crack tip are obtained. The type of mixed loading is specified by the mixity parameter which is varying from 0 (this type of loading corresponds to pure shear) to 1 (the loading corresponds to tensile loading). The analytical presentation of the stress and the creep strain rate fields is found for all values of the mixity parameter. It is shown that the stress field consists of different regions inside which the stress components are determined by different formulae. The boundaries of the regions are found numerically. The comparison of the analytical solution with the numeric solution obtained for the power-law material for large values of the exponent $n$ is given.

Keywords: mixed mode (mode I + mode II) loading, asymptotical analysis, the near crack-tip stress field, analytical solution, analytical presentation of the stress field near the crack tip.

Received 11/XII/2013;

received in revised form $17 / \mathrm{II} / 2014$;

accepted $21 / \mathrm{II} / 2014$.

ISSN: 2310-7081 (online), 1991-8615 (print); doi: http://dx.doi.org/10.14498/vsgtu1297 (C) 2014 Samara State Technical University.

Citation: L. V. Stepanova, E. M. Adylina, "Stress Field Near the Mixed Mode Crack Tip under Plane Stress Conditions", Vestn. Samar. Gos. Tekhn. Univ., Ser. Fiz.-Mat. Nauki [J. Samara State Tech. Univ., Ser. Phys. \& Math. Sci.], 2014, no. 1 (34), pp. $109-124$. doi: 10.14498/vsgtu1297. (In Russian)

Authors Details: Larisa V. Stepanova (Dr. Phys. \& Math. Sci.), Professor, Dept. of Mathematical Modelling in Mechanics. Ekaterina M. Adylina, Postgraduate Student, Dept. of Mathematical Modelling in Mechanics.

E-mail addresses: 1st@ssu.samara.ru (L.V. Stepanova, Corresponding author), kateadulina@mail.ru (E.M. Adylina) 